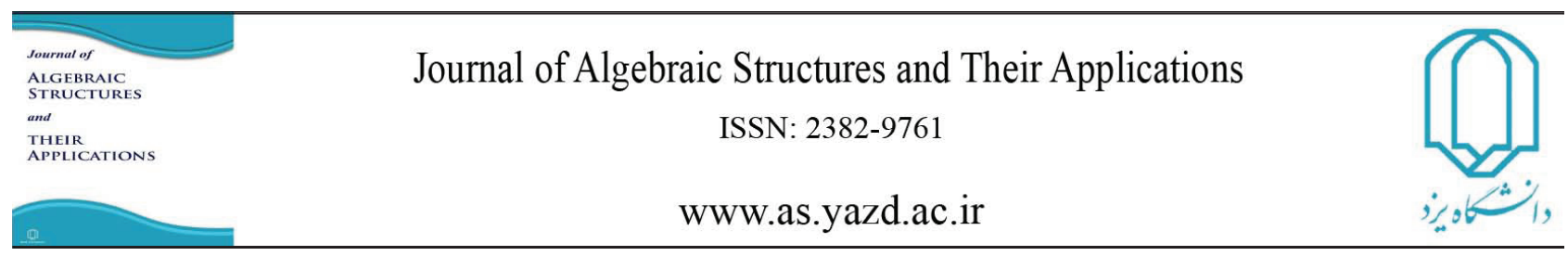

Algebraic Structures and Their Applications Vol. 4 No. 1 ( 2017 ) pp 45-52.

\title{
ON TWO-DIMENSIONAL CAYLEY GRAPHS
}

\author{
ALI BEHTOEI* AND YASER GOLKHANDY POUR
}

Communicated by M.A. Iranmanesh

\begin{abstract}
A subset $W$ of the vertices of a graph $G$ is a resolving set for $G$ when for each pair of distinct vertices $u, v \in V(G)$ there exists $w \in W$ such that $d(u, w) \neq d(v, w)$. The cardinality of a minimum resolving set for $G$ is the metric dimension of $G$. This concept has applications in many diverse areas including network discovery, robot navigation, image processing, combinatorial search and optimization. The problem of finding metric dimension is NP-complete for general graphs but the metric dimension of trees can be obtained using a polynomial time algorithm. In this paper, we investigate the metric dimension of Cayley graphs on dihedral groups and we characterize a family of them.
\end{abstract}

\section{INTRODUCTION}

Let $\Gamma=(V, E)$ be a simple and connected graph with vertex set $V$ and edge set $E$. The distance between two vertices $x, y \in V$ is the length of a shortest path between them and is denoted by $d(x, y)$. If $d(x, y)=1$, then for convenient we write $x \sim y$. The neighborhood of $x$ is $N(x)=\{y: x \sim y\}$. A walk consists of an alternating sequence of vertices and edges http://dx.doi.org/10.29252/asta.4.1.43

MSC(2010): Primary: 05C25. Secondary: 05C75, $20 \mathrm{~B} 10$.

Keywords: Resolving set, Metric dimension, Cayley graph, Dihedral group.

Received: 20 September 2017, Accepted: 10 February 2018

* Corresponding author

(C) 2017 Yazd University. 
consecutive elements of which are incident, that begins and ends with a vertex. A walk is said to be closed if its endpoints are the same. The length of a walk is the number of its edges. An odd walk is a walk whose length is an odd number. It is well known that a graph is bipartite if and only if it does not contain any odd walk. A matching or independent edge set in a graph is a set of edges without common vertices. In a graph of even ordr $n=|V(G)|$, each matching with $\frac{n}{2}$ edges is called a perfect matching. For an ordered subset $W=\left\{w_{1}, w_{2}, \ldots, w_{k}\right\}$ of vertices and a vertex $v \in V$, the $k$-vector $r(v \mid W):=\left(d\left(v, w_{1}\right), d\left(v, w_{2}\right), \ldots, d\left(v, w_{k}\right)\right)$ is called the metric representation of $v$ with respect to $W$. The set $W$ is called a resolving set for $\Gamma$ if distinct vertices of $\Gamma$ have distinct representations with respect to $W$. Each minimum resolving set is a basis and the metric dimension of $\Gamma$, $\operatorname{dim}_{M}(\Gamma)$, is the cardinality of a basis for $\Gamma$. These concepts were introduced by Slater in 1975 when he was working with U.S. Sonar and Coast Guard Loran stations and he described the usefulness of these concepts, (see [15]). Independently, Harary and Melter discovered these concepts, (see [7]). This concept has applications in many areas including network discovery and verification (see [2]), robot

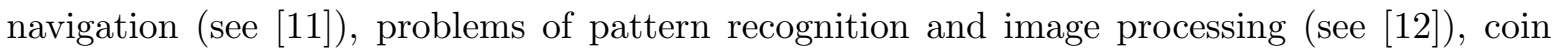
weighing problems (see [14]), strategies for the Mastermind game (see [5]), combinatorial search and optimization (see [14]). Finding families of graphs with constant metric dimension or characterizing $n$-vertex graphs with a specified metric dimension are fascinating problems and attracts the attention of many researchers. The problem of finding metric dimension is NP-Complete for general graphs but the metric dimension of trees can be obtained using a polynomial time algorithm. It is not hard to see that for each $n$-vertex graph $\Gamma$ we have $1 \leq \operatorname{dim}_{M}(\Gamma) \leq n-1$. Chartrand et al. in [6] proved that for $n \geq 2, \operatorname{dim}_{M}(\Gamma)=n-1$ if and only if $\Gamma$ is the complete graph $K_{n}$. The metric dimension of each complete $t$-partite graph with $n$ vertices is $n-t$. They also provided a characterization of graphs of order $n$ with metric dimension $n-2$, (see [6] ). Graphs of order $n$ with metric dimension $n-3$ are characterized in [9]. Khuller et al. (see [I]]) and Chartrand et al. (see [6]) proved that $\operatorname{dim}_{M}(\Gamma)=1$ if and only if $\Gamma$ is a path $P_{n}$. Salman et al. studied this parameter for the Cayley graphs on cyclic groups, (see [1]:3]). Imran studied the metric dimension of barycentric subdivision of Cayley graphs in $[8]$. Each cycle graph $C_{n}$ is a 2-dimensional graph $\left(\operatorname{dim}_{M}\left(C_{n}\right)=2\right)$. All of 2-trees with metric dimension two are characterized in [3]. Moreover, in [II] and [16] some properties of 2-dimensional graphs are obtained.

Theorem 1.1. [II] Let $\Gamma$ be a 2-dimensional graph. If $\{u, v\}$ is a basis for $\Gamma$, then

(1) there is a unique shortest path $P$ between $u$ and $v$,

(2) the degrees of $u$ and $v$ are at most three,

(3) the degree of each internal vertex on $P$ is at most five. 
The Möbius Ladder graph $M_{n}$ is a cubic circulant graph with an even number $n$ of vertices formed from an $n$-cycle by connecting opposite pairs of vertices in the cycle. For the metric dimension of Möbius Ladders we have the following result.

Theorem 1.2. [I] Let $n \geq 8$ be an even number. The metric dimension of each Mobius Ladder $M_{n}$ is 3 or 4. Specially, $\operatorname{dim}_{M}\left(M_{n}\right)=3$ when $n \equiv 2(\bmod 8)$.

Cáceres et al. studied the metric dimension of the Cartesian product of graphs. Recall that the Cartesian product of two graphs $G_{1}$ and $G_{2}$, denoted by $G_{1} \times G_{2}$, is a graph with vertex set $V\left(G_{1}\right) \times V\left(G_{2}\right):=\left\{(u, v): u \in V\left(G_{1}\right), v \in V\left(G_{2}\right)\right\}$, in which $(u, v)$ is adjacent to $\left(u^{\prime}, v^{\prime}\right)$ whenever $u=u^{\prime}$ and $v v^{\prime} \in E\left(G_{2}\right)$, or $v=v^{\prime}$ and $u u^{\prime} \in E\left(G_{1}\right)$.

Theorem 1.3. [4] Let $P_{m}$ be a path on $m \geq 2$ vertices and $C_{n}$ be a cycle on $n \geq 3$ vertices. Then the metric dimension of each prism $P_{m} \times C_{n}$ is given by

$$
\operatorname{dim}_{M}\left(P_{m} \times C_{n}\right)= \begin{cases}2 & n \text { odd }, \\ 3 & n \text { even } .\end{cases}
$$

Let $G$ be a group and let $S$ be a subset of $G$ that is closed under taking inverse and does not contain the identity element, say $e$. Recall that the Cayley graph $\operatorname{Cay}(G, S)$ is a graph whose vertex set is $G$ and two vertices $u$ and $v$ are adjacent in it when $u v^{-1} \in S$. Since $S$ is inverse-closed $\left(S=S^{-1}\right)$ and does not contain the identity, $\operatorname{Cay}(G, S)$ is a simple graph. It is well known that $\operatorname{Cay}(G, S)$ is a connected graph if and only if $S$ is a generating set for $G$. Since $\operatorname{Cay}(G, S)$ is $|S|$-regular, part (2) of Theorem $\llbracket$ d directly implies the following result.

Corollary 1. If $S$ is a subset of $D_{2 n}$ such that $e \notin S=S^{-1}$ and $|S| \geq 4$, then we have

$$
\operatorname{dim}_{M}\left(\operatorname{Cay}\left(D_{2 n}, S\right)\right) \geq 3 \text {. }
$$

For more results in this subject or related subjects see [6], [8] and [IT]. In this paper, we study the metric dimension of Cayley graphs on dihedral groups and we characterize all of Cayley graphs on dihedral groups whose metric dimension is two.

\section{Main results}

At first, we provide two lemmas on dihedral groups and a sharp lower bound for the metric dimension of 3-regular bipartite graphs which will be frequently used in the sequel.

Lemma 2.1. The subset $\left\{a^{i} b, a^{j} b\right\}$ is a generating set for dihedral group $D_{2 n}=\langle a, b| a^{n}=$ $\left.b^{2}=(a b)^{2}=e\right\rangle$ if and only if $\operatorname{gcd}(n, i-j)=1$.

Proof. It is strightforward to see that the subgroup generated by these elements is given by

$$
\left\langle a^{i} b, a^{j} b\right\rangle=\left\{a^{(i-j) t}, a^{(i-j) t+i} b, a^{(i-j) t+j} b \mid t \in \mathbb{Z}\right\} .
$$


Now since we have $a \in\left\langle a^{i-j}\right\rangle$ if and only if $\operatorname{gcd}(n, i-j)=1$, the result follows.

Lemma 2.2. If $4 \mid n$ and $\operatorname{gcd}(i-j, n)=2$, then $\left\{a^{\frac{n}{2}}, a^{i} b, a^{j} b\right\}$ is not a generating set for $D_{2 n}$.

Proof. Since $\left\langle a^{i-j}\right\rangle$ and $\left\langle a^{2}\right\rangle$ are two cyclic subgroups of order $\frac{n}{2}$ in the cyclic group $\langle a\rangle$, we have $\left\langle a^{2}\right\rangle=\left\langle a^{i-j}\right\rangle \subseteq\left\langle\left\{a^{\frac{n}{2}}, a^{i} b, a^{j} b\right\}\right\rangle$. Since $4 \mid n$ we have $a^{\frac{n}{2}} \in\left\langle a^{2}\right\rangle$ and hence, $\left\langle\left\{a^{\frac{n}{2}}, a^{i} b, a^{j} b\right\}\right\rangle=$ $\left\langle\left\{a^{i} b, a^{j} b\right\}\right\rangle$. Now the result follows from Lemma [2.].

Lemma 2.3. Let $\Gamma$ be a 3-regular bipartite graph on $n$ vertices. Then $\operatorname{dim}_{M}(\Gamma) \geq 3$.

Proof. Since $\Gamma$ is not a path, $\operatorname{dim}_{M}(\Gamma)$ is at least two. Suppose that $\operatorname{dim}_{M}(\Gamma)=2$ and let $W=\{u, v\}$ be a resolving set for $\Gamma$. Assume that $d(u, v)=d$ and $N(u)=\left\{u_{1}, u_{2}, u_{3}\right\}$. It is easy to see that $d\left(u_{i}, v\right) \in\{d-1, d, d+1\}$, for each $1 \leq i \leq 3$. If there exist $1 \leq i<j \leq 3$ such that $d\left(u_{i}, v\right)=d\left(u_{j}, v\right)$, then $r\left(u_{i} \mid W\right)=r\left(u_{j} \mid W\right)$, which is a contradiction. Hence, without loss of generality, we can assume that

$$
d\left(u_{1}, v\right)=d-1, d\left(u_{2}, v\right)=d, d\left(u_{3}, v\right)=d+1 .
$$

Let $\sigma_{1}$ be a (shortest) path between two vertices $u$ and $v$ of lengh $d$, and $\sigma_{2}$ be a (shortest) path between two vertices $u_{2}$ and $v$. Two paths $\sigma_{1}$ and $\sigma_{2}$ using the edge $u u_{2}$ produce an old closed walk of lengh $2 d+1$ in $\Gamma$ which contradicts the fact that $\Gamma$ is a bipartite graph. For the sharpness of this bound, consider the hypercube $Q_{3}=K_{2} \times K_{2} \times K_{2}$.

In Theorem 2.4 we characterize all of Cayley graphs on dihedral groups whose metric dimension is two. Recall that the center of $D_{2 n}$ is $\left\langle a^{\frac{n}{2}}\right\rangle$ when $n$ is even, otherwise it is the trivial subgroup $\{e\}$.

Theorem 2.4. Let $S$ be a generating subset of $D_{2 n}=\left\langle a, b \mid a^{n}=b^{2}=(a b)^{2}=e\right\rangle$ such that $e \notin S=S^{-1}$. Then we have $\operatorname{dim}_{M}\left(\operatorname{Cay}\left(D_{2 n}, S\right)\right)=2$ if and only if one of the following cases occurs.

a) $n=2$ and $S \in\{\{a, b\},\{a, a b\},\{b, a b\}\}$,

b) $n \geq 3$ and $S=\left\{a^{i} b, a^{j} b\right\}$ with $\operatorname{gcd}(i-j, n)=1$,

c) $n \geq 3$ is odd and $S=\left\{a^{i}, a^{-i}, a^{j} b\right\}$ with $\operatorname{gcd}(i, n)=1$ and $j \in\{1,2, \ldots, n\}$.

Proof. First suppose that $\operatorname{dim}_{M}\left(\operatorname{Cay}\left(D_{2 n}, S\right)\right)=2$. Since $D_{2 n}$ is not a cyclic group, we have $|S| \geq 2$. Also, $\operatorname{Cay}\left(D_{2 n}, S\right)$ is $|S|$-regular and part (2) of Theorem ㄸ. implies that $|S| \leq 3$. Thus, $2 \leq|S| \leq 3$. If $|S|=2$, then $\operatorname{Cay}\left(D_{2 n}, S\right)$ is a connected 2-regular graph (a cycle) and $\operatorname{dim}_{M}\left(\operatorname{Cay}\left(D_{2 n}, S\right)\right)=2$. Moreover, with the assumption $S=\{x, y\}$, since $S=S^{-1}$ and $D_{2 n}$ is not cyclic, we have $y \neq x^{-1}$ and $x^{2}=y^{2}=e$. If $S=\left\{a^{\frac{n}{2}}, a^{j} b\right\}$ for some 
$1 \leq j \leq n$, then the condition $D_{2 n}=\langle S\rangle$ implies that $n=2, D_{2 n}=D_{4}=\{e, a, b, a b\}$ and $S \in\{\{a, b\},\{a, a b\},\{b, a b\}\}$ which provides the case (a). Otherwise, $S=\left\{a^{i} b, a^{j} b\right\}$ and using Lemma [.] we have $\operatorname{gcd}(i-j, n)=1$ and this provides the case (b). Now we can assume that $|S|=3$. Since $S$ is a generating set and $e \notin S=S^{-1}$, we consider the following cases.

Case 1. $S=\left\{a^{i}, a^{-i}, a^{j} b\right\}$.

Since $\left(a^{j} b\right)\left(a^{i}\right)^{t}\left(a^{j} b\right)=a^{-i t}$, the order of $a^{i}$ is $\frac{n}{\operatorname{gcd}(i, n)}$ and $S$ is a generating set, we have $\operatorname{gcd}(i, n)=1$. Thus $o\left(a^{i}\right)=n$ and vertices $a^{n i}, a^{(n-1) i}, \ldots, a^{2 i}, a^{i}$ induce an $n$-cycle in $\operatorname{Cay}\left(D_{2 n}, S\right)$. Since $a^{j} \in\left\langle a^{i}\right\rangle$, there exists $k \in\{1,2, \ldots, n\}$ such that $a^{j}=a^{k i}$. Therefore $n$ vertices

$$
a^{k i} b, a^{(k+1) i} b, \ldots, a^{(k+n-2) i} b, a^{(k+n-1) i} b
$$

induce another cycle in $\operatorname{Cay}\left(D_{2 n}, S\right)$. Now for each $1 \leq \ell \leq n$ let $M_{\ell}=\left\{a^{\ell i}, a^{(k+n-\ell) i} b\right\}$. Note that $a^{n i}=e$ and $M_{s} \cap M_{k}=\emptyset$ for each $s \neq k$. Since $a^{\ell i}\left(a^{(k+n-\ell) i} b\right)^{-1}=a^{k i} b=a^{j} b \in S$, two vertices $a^{\ell i}$ and $a^{(k+n-\ell) i} b$ are adjacent in $\operatorname{Cay}\left(D_{2 n}, S\right)$. Thus, the edges $M_{1}, M_{2}, \ldots, M_{n}$ provide a perfect matching in $\operatorname{Cay}\left(D_{2 n}, S\right)$. Consequently, $\operatorname{Cay}\left(D_{2 n}, S\right)$ is isomorphic to $P_{2} \times C_{n}$. Now Theorem 4.3 implies that $\operatorname{dim}_{M}\left(\operatorname{Cay}\left(D_{2 n}, S\right)\right)=2$ if and only if $n$ is odd. This provides the case (c). In the sequel we will show that other cases for $S$ are impossimble and they will cause some contradictions.

Case 2. $S=\left\{a^{n / 2}, a^{i} b, a^{j} b\right\}$ where $n$ is an even number.

Let $x=a^{i} b$ and $y=a^{j} b$. Since $a^{n / 2}$ is in the center of $D_{2 n}$ and $o\left(a^{\frac{n}{2}}\right)=2$, we have $\langle S\rangle=\left\langle a^{i} b, a^{j} b\right\rangle \cup a^{n / 2}\left\langle a^{i} b, a^{j} b\right\rangle$. Hence, $a \in\left\langle a^{i} b, a^{j} b\right\rangle$ or $a \in a^{n / 2}\left\langle a^{i} b, a^{j} b\right\rangle$. Note that

$$
\left|\left\langle a^{n / 2}, a^{i} b\right\rangle\right|=\left|\left\langle a^{n / 2}, a^{j} b\right\rangle\right|=4
$$

Thus, $a \notin\left\langle a^{n / 2}, a^{i} b\right\rangle$ and $a \notin\left\langle a^{n / 2}, a^{j} b\right\rangle$.

Subcase 2.1. $a \in\left\langle a^{i} b, a^{j} b\right\rangle$.

In this case, using Lemma 2.0 we have $\operatorname{gcd}(i-j, n)=1$. Thus, $o(x y)=o\left(a^{i-j}\right)=n$ and $\operatorname{Cay}\left(D_{2 n}, S\right)$ contains a Hamiltonian cycle (on $2 n$ vertices) as below.

$$
e \sim y \sim x y \sim y x y \sim(x y)^{2} \sim y(x y)^{2} \sim \ldots \sim y(x y)^{n-1} \sim(x y)^{n}=e .
$$

For each divisor $d$ of $n$ the cyclic group $\mathbb{Z}_{n}$ has unique cyclic subgroup of order $d$. Since $\left\langle a^{i-j}\right\rangle=\langle a\rangle$ and $\left|\left\langle a^{(i-j) \frac{n}{2}}\right\rangle\right|=\left|\left\langle a^{\frac{n}{2}}\right\rangle\right|=2$, we have $a^{n / 2}=\left(a^{i-j}\right)^{n / 2}$. For each $1 \leq \ell \leq \frac{n}{2}$ let $M_{\ell}=\left\{(x y)^{\ell},(x y)^{\ell+n / 2}\right\}$ and $T_{\ell}=\left\{y(x y)^{\ell}, y(x y)^{\ell+n / 2}\right\}$. Note that $M_{s} \neq M_{k}$ and $T_{s} \neq T_{k}$ for each $s \neq k$. Also, each $M_{\ell}$ is an edge in $\operatorname{Cay}\left(D_{2 n}, S\right)$ because

$$
(x y)^{\ell+n / 2}(x y)^{-\ell}=(x y)^{n / 2}=\left(a^{i-j}\right)^{n / 2}=a^{n / 2} \in S
$$


Thus, $\left\{M_{1}, M_{2}, \ldots, M_{\frac{n}{2}}\right\}$ is a matching in $X\left(D_{n}, S\right)$. Similarly, $\left\{T_{1}, T_{2}, \ldots, T_{\frac{n}{2}}\right\}$ is a matching and hence, $\left\{M_{1}, M_{2}, \ldots, M_{\frac{n}{2}}, T_{1}, T_{2}, \ldots, T_{\frac{n}{2}}\right\}$ provides a perfect matching for $\operatorname{Cay}\left(D_{2 n}, S\right)$. Therefore, we have a cycle on $2 n$ vertices in which its opposite pairs of vertices are adjacent (see Figure प (i)). This implies that $\operatorname{Cay}\left(D_{2 n}, S\right)$ is a Möbius Ladder and by Theorem ए.2, $\operatorname{dim}_{M}\left(\operatorname{Cay}\left(D_{2 n}, S\right)\right)$ is 3 or 4 , which is a contradiction.

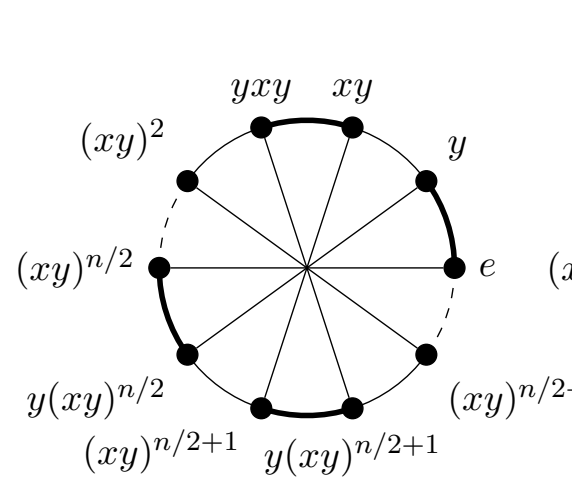

$(i)$

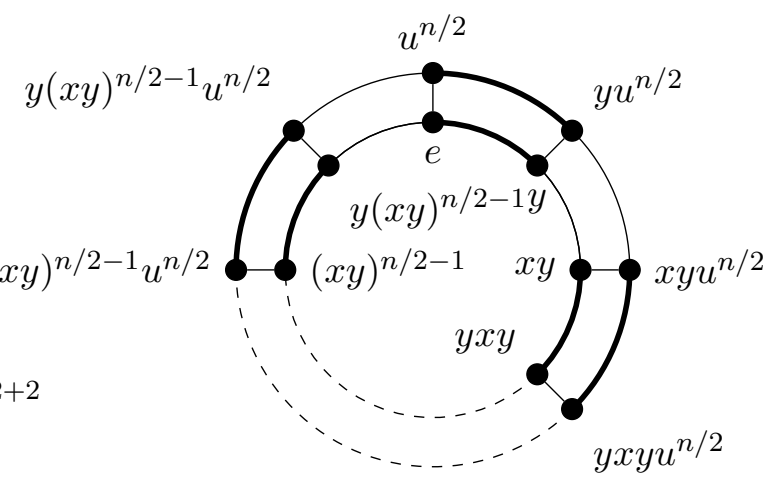

(ii)

Figure 1. (i) A Möbius Ladder graph, and (ii) the Cartesian product $P_{2} \times C_{n}$.

Subcase 2.2. $a \in a^{n / 2}\left\langle a^{i} b, a^{j} b\right\rangle$.

In this case, there exists $k \in \mathbb{Z}$ such that $a=a^{\frac{n}{2}}\left(a^{i-j}\right)^{k}$. Hence, $a^{\frac{n}{2}+1} \in\left\langle a^{i-j}\right\rangle$ and $a^{2}=$ $\left(a^{\frac{n}{2}+1}\right)^{2} \in\left\langle a^{i-j}\right\rangle$. Thus, $\left|\left\langle a^{i-j}\right\rangle\right| \geq\left|\left\langle a^{2}\right\rangle\right|=\frac{n}{2}$. Hence, $o\left(a^{i-j}\right)=\frac{n}{2}$ or $o\left(a^{i-j}\right)=n$. The situation $o\left(a^{i-j}\right)=n$ is considered in Subcase 2.1 and we can assume that $o(x y)=o\left(a^{i-j}\right)=n / 2$. Therefore, $\operatorname{Cay}\left(D_{2 n}, S\right)$ contains two $n$-cycles as below.

$$
\begin{gathered}
e \sim y \sim x y \sim y x y \sim(x y)^{2} \sim y(x y)^{2} \sim \ldots \sim y(x y)^{n / 2-1} \sim(x y)^{n / 2}=e, \\
a^{n / 2} \sim y a^{n / 2} \sim(x y) a^{n / 2} \sim y(x y) a^{n / 2} \sim(x y)^{2} a^{n / 2} \sim \ldots \sim(x y)^{n / 2} a^{n / 2}=a^{n / 2} .
\end{gathered}
$$

The fact $o(x y)=n / 2$ implies that $n$ vertices appeared in each cycle are distinct. Also, using the appearence of $b$, it is easy to see that $(x y)^{t} \neq y(x y)^{s} a^{\frac{n}{2}}$ and $y(x y)^{t} \neq(x y)^{s} a^{\frac{n}{2}}$ for each $t, s \in \mathbb{Z}$. If there exist $t, s \in \mathbb{Z}$ such that $(x y)^{t}=(x y)^{s} a^{\frac{n}{2}}$ or $y(x y)^{t}=y(x y)^{s} a^{\frac{n}{2}}$, then $a^{\frac{n}{2}}=\left(a^{i-j}\right)^{(t-s)} \in\left\langle a^{i-j}\right\rangle=\left\langle a^{2}\right\rangle$. Thus, $4 \mid n$ which is a contradiction (see Lemma [2.2). Therefore, all of $2 n$ vertices appeared in these cycles are distinct. Since $a^{\frac{n}{2}} \in S$, corresponding vertices of two cycles are adjacent (see Figure $\mathbf{U}(\mathrm{ii})$ ). Hence, $\operatorname{Cay}\left(D_{2 n}, S\right)$ is isomorphic to $P_{2} \times C_{n}$ and Theorem ㄴ.3 implies that $\operatorname{dim}_{M}\left(\operatorname{Cay}\left(D_{2 n}, S\right)\right)=3$. This is a contradiction.

Case 3. $S=\left\{a^{i} b, a^{j} b, a^{t} b\right\}$. 
Let $H=\langle a\rangle$ and hence, $V\left(\operatorname{Cay}\left(D_{2 n}, S\right)\right)=H \cup H b$. If $a^{s}, a^{t} \in H$, then $a^{s} a^{-t}=a^{s-t} \notin S$. Thus, the subset $H$ of vertices induces an independent set in $\operatorname{Cay}\left(D_{2 n}, S\right)$. Similarly, $H b$ is an independent set. Consequently, $\operatorname{Cay}\left(D_{2 n}, S\right)$ is a 3-regular bipartite graph on $2 n$ vertices. Now Lemma $\left[2.3\right.$ implies that $\operatorname{dim}_{M}\left(\operatorname{Cay}\left(D_{2 n}, S\right)\right)$ is at least three, a contradiction.

For the converse suppose that one of the cases (a), (b) or (c) occurs. If case (a) or case (b) occurs, then using Lemma [2.], the graph $\operatorname{Cay}\left(D_{2 n}, S\right)$ is 2-regular and connected. Thus, it is is a cycle and its metric dimension is two. If case (c) occurs, then using Case 1 in this proof, we see that $\operatorname{Cay}\left(D_{2 n}, S\right)$ is isomorphic to $P_{2} \times C_{n}$. Now, Theorem 4.3 implies that $\operatorname{dim}_{M}\left(\operatorname{Cay}\left(D_{2 n}, S\right)\right)=2$ because $n$ is odd. This completes the proof.

\section{AcKnowledgments}

We would like to express our deepest gratitude to the referees for their invaluable comments and suggestions which improve the quality of this paper.

\section{REFERENCES}

[1] M. Ali, G. Ali, M. Imran, A.Q. Baig, M.K.F. Shafiq, On the metric dimension of Mobius ladders, Ars Combin. 105 (2012) 103-410.

[2] Z. Beerliova, F. Eberhard, T. Erlebach, A. Hall, M. Hoffmann, M. Mihalák, L.S. Ram, Network dicovery and verification, IEEE J. Sel. Areas Commun. 24 (2006) 2168-2181.

[3] A. Behtoei, A. Davoodi, M. Jannesari, B. Omoomi, A characterization of some graphs with metric dimension two, Discrete Math. Algorithm. Appl. (2017) DOI: 10.1142/S1793830917500276.

[4] J. Caceres, C. Hernando, M. Mora, I.M. Pelayo, M.L. Puertas, C. Seara, D.R. Wood, On the metric dimension of cartesian products of graphs, SIAM J. Discrete Math. 21 (2007) 423-441.

[5] V. Chvátal, Mastermind, Combinatorica, 3 (1983) 325-329 .

[6] G. Chartrand, L. Eroh, M.A. Johnson, O.R. Ollermann, Resolvability in graphs and the metric dimension of a graph, Discrete Appl. Math. 105 (2000) 99-113.

[7] F. Harary, R.A. Melter, On the metric dimension of a graph, Ars Combin. 2 (1976) 191-195.

[8] M. Imran, On the metric dimension of barycentric subdivision of Cayley graphs, Acta Math. Appl. Sin. Engl. Ser. 32 (2016) 1067-1072.

[9] M. Janessari, B. Omoomi, Characterization of n-vertex graphs with metric dimension $n-3$, Math. Bohem. 139 (2014) 1-23.

[10] A. Kelenc, D. Kuziak, A. Taranenko, I.G. Yero, Mixed metric dimension of graphs, Appl. Math. Comput. 314 (2017) 429-438.

[11] S. Khuller, B. Raghavachari, A. Rosenfeld, Landmarks in graphs, Discrete Appl. Math. 70 (1996) $217-229$.

[12] R.A. Melter, I. Tomescu, Metric bases in digital geometry, Comput. Gr. Image Process. 25 (1984) $113-121$.

[13] M. Salman, I. Javaid, M.A. Chaudhry, Resolvability in circulant graphs, Acta Math. Sin. (Engl. Ser.) 29 (2012) 1851-1864.

[14] A. Sebo, E. Tannier, On metric generators of graphs, Math. Oper. Res. 29 (2004) 383-393. 
[15] P.J. Slater, Leaves of trees, Congr. Numer. 14 (1975) 549-559.

[16] G. Sudhakara, A.R. Hemanth Kumar, Graphs with metric dimension two-a characterization, World Academy of Science, Engineering and Technology. 36 (2009) 621-626.

\author{
Ali Behtoei \\ Department of Mathematics, Faculty of Science, \\ Imam Khomeini International University, \\ Qazvin, Iran, POBox: 34148 - 96818, \\ a.behtoei@sci.ikiu.ac.ir

\section{Yaser Golkhandy Pour} \\ Department of Mathematics, Faculty of Science, \\ Imam Khomeini International University, \\ Qazvin, Iran, POBox: 34148 - 96818, \\ y.golkhandypour@edu.ikiu.ac.ir
}

